6.Q Liang,H Liu,H Xing,et al.High-resolution mass spectrometry for exploring metabolic signatures of sepsis-induced acute kidney injury[J].Rsc Advances,2016,6(36):29863-29868.

7.Q Liang, H Liu, T Zhang,et al.Metabolomics-based screening of salivary biomarkers for early diagnosis of Alzheimer's disease[J]. Rsc Advances,2015,5(116):96074-96079.

8.Guoye Ji,Bin Wang,Jiang Du,et al.Effects of hemoperfusion at different time on blood IL-6 and TNF-a levels in rabbits with sepsis[J]. Modern Medical Journal,2011,39(2):129-133.

\title{
DOI 10.22448/AMJ.2017.3.59-60
}

\section{FIVE 1 TECHNOLOGY FINDINGS IN THE PERMEATION RATE OF APPLICATION AT ACUPOINT}

\author{
Rui Wang1, Qun Feng, Yanhong Wang, Jing Yang and Yongji Li* \\ Heilongjiang University of Chinese Medicine, Harbin, Heilongjiang
}

1First author: A/Prof. Rui Wang, Heilongjiang University of Chinese Medicine, Harbin, Heilongjiang, 150040. Tel: 0451-87266893, E-mail:wrdx@sina.com

*Correspondence author: Prof. Yongji Li, Heilongjiang University of Chinese Medicine, Harbin, Heilongjiang, 150040. Tel: 0451-87266893; E-mail: liyongji2009@163.com

Abstract Objective The acupoint is an important role in the traditional Chinese medicine. However, it is unclear that the potential mechanism of acupoint effect, and whether had other characters. We conjecture that acupoint application can enhance the permeation rate of skin.

Methods This study, we through application fluorochrome at Zusanli (ST 36) and general site of rabbits and employ Fluorescence in vivo Endomicroscopy (FIVE 1) to obtain the images of connective tissue under the skin. And compare the fluorescence images of acupoint and general site in 5, 10 and $15 \mathrm{~min}$.

Results the acupoint group was more quickly to achieve the fluorescence intensity which could to observe the connective tissue.

Discussion It is revealed that application at Zusanli (ST 36) could improve the permeation rate of fluorochrome.

Key words: acupoint application, Zusanli (ST 36), FIVE 1

Acupoint is an important role in the traditional Chinese medicine, and it is the basis of manipulation and acupuncture. It has been employed to treat various diseases more than 2000 years in East Asia by inserting and manipulating in acupoints1. Acupuncture has become an important complementary and alternative medicine treatment in western countries2. However, the potential mechanism of acupoint has been difficult to illuminate. Thus, we conjectured that application at acupoint could change the permeation rate of skin.

Objective To assess the permeation rates of acupoint and general site employ the FIVE 1, and it will reveal the advantage of acupoint application.

Materials and methods At first, injected anesthetics in ear marginal vein to anesthetize. Random choice five rabbits as test group to point application fluorochrome at Zusanli (ST 36), and the others five rabbits as control group were application fluorochrome at the general site which was in the left $2 \mathrm{~cm}$ of ST 36. Then FIVE 1 probe was pierced at the subcutaneous tissue to observe the level of permeability of fluorochrome for 5, 10 and $15 \mathrm{~min}$.

Results and discussion After 5 min, the images of connective tissue were shown as Fig. 2. The fluorescence intensity of Zusanli (ST 36) group was enough to observe the characteristic of connective tissue, and the general site group was too dark to be able to observe the connective tissue.
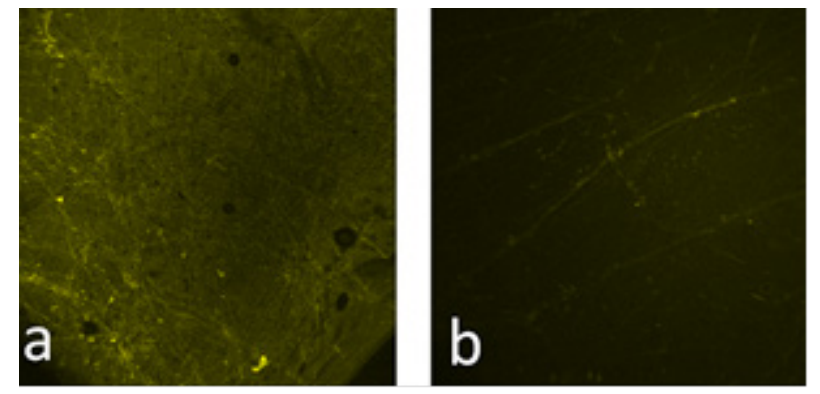

Fig. 2 The images of connective tissue. a was the Zusanli (ST 36) image; b was the general site group image.

As shown in Fig. 3. After 10 min, the connective tissue of two groups was all clear, but Zusanli (ST 36) group was more brightly than control group. 

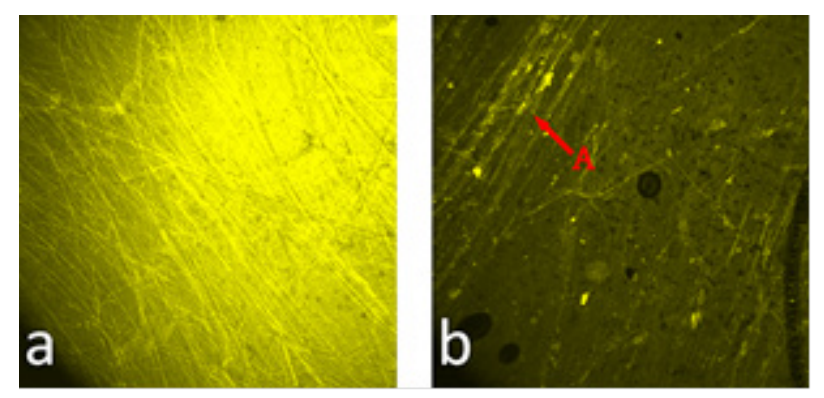

Fig. 3 The images of connective tissue. a was the Zusanli (ST 36) image; b was the general site group image. A was the fiber which had been stained

As a result, compared the images of test group and control group, the test group was more quickly to achieve the fluorescence intensity which could to observed the connective tissue. It was revealed that application at Zusanli (ST 36) could improve the permeation rate. However, the mechanism was not clear, and due to samples capacity was small, we should be studied further more.

\title{
References:
}

1. Jang, J.H., Lee, D.J., Bae, C.H., Ha, K.T., Kwon, S., Park, H.J., Hahm, D.H., Lee, H., Kim, S. Changes in small intestinal motility and related hormones by acupuncture stimulation at zusanli (st 36) in mice// Chin J Integr Med. - 2017. - 23 (3). - J.215-220.

2. Kim, J., Kang, D.I., Soh, K.S., Kim, S. Analysis on postmortem tissues at acupuncture points in the image datasets of visible human project// J Altern Complement Med. - 2012. - 18 (2). - J.120-129.

\section{UDC 616.25:611-08-25 DOI 10.22448/AMJ.2017.3.60-61}

\section{MODELS OF EXPERIMENTAL PLEURITE APPLICABLE ON LABORATORY ANIMALS}

\author{
Semenov D.A.
}

\author{
Amur State Medical Academy, Blagoveshchensk, Russian Federation
}

Abstract Currently, there are no models that fully correspond to human inflammatory diseases. The model in laboratory animals should meet several basic requirements: 1 . It should provide detailed research and quantification of various parameters. 2. It must be reproducible and ensure that a sufficient number of experiments are performed for statistical analysis.

Key words: experimental pleurisy, irritant, cell and cell-free exudate, inflammation

Separation into acute and chronic inflammation is important in the development or interpretation of animal models. For a histologist, acute inflammation is determined by the presence of polymorphonuclear leukocytes. Chronic inflammation, in contrast, is characterized by the presence of mononuclear cells (macrophages and lymphocytes).

Inflammation, which in its outward manifestations is always the same, can be the result of the activation of many different chemical mediator systems. To this end, two types of inflammation are used: immune and non-immune.

The introduction of irritants into closed body cavities leads to the formation of liquid exudate with a large content of cells. The most suitable cavity for studying the inflammatory reaction was the pleural cavity.

It was revealed that intrapleural injection of silver nitrate, tetracycline derivatives, talc and mitoxantrone results in acute exudative pleural effusion within 12 hours [3].

Irrantant is injected into the pleural cavity of anesthetized rats. Typically, the skin on one side of the chest is pulled and the cut on the III and IV ribs is made with the scalpel blade. Using a thick needle, $0.1 \mathrm{ml}$ of irritant (usually carrageenan) is injected into the pleural cavity. The test compound is administered before intrapleural injection of the irritant. Animals are killed in 4, 6, 24, 48 hours, remove the exudate, measure its volume. Centrifuge, obtaining an acellular composition (pinpointing mediators, histamine, kinin) and cells (perform a general and differentiated cell count in vitro). Various irrigants are used, including terpentine (to its disadvantages is the destruction of migrating leukocytes) and carrageenan. Carrageenan pleurisy provides the secretion of histamine, 5-hydroxytryptamine, kinins and prostaglandins. Experimental models of pleurisy reveal cyclooxygenase inhibitors. Possible inflammation, causing inflammation, the closest to the clinical, is calcium pyrophosphate dihydrate. Its crystals cause a non-immune reaction. The response to steroid and non-steroid drugs is comparable to the response to the carrageenan pleurisy model. The merits of this system include the fact that comparing the findings with the results of this test allows you to give a conclusion about the effects of drugs on the complement system. In the models of pleurisy, steroid and non-steroid preparations can be determined by their effect on 24-hour damage. The merits of the pleurisy model include its easy objectification and accessibility as an exudate for biochemical analysis, as well as cells for morphological research. The drawback of the model is the need to prepare a group of animals for each time interval, which is difficult for the operator and increases the cost of the experiment [1,4]. 